\title{
Dosage des IgG du lait de vache par immunodiffusion radiale semi-automatisée, pour la détection du colostrum, des laits de mammites ou de fin de gestation. I. Mise au point du dosage
}

\author{
D Levieux \\ INRA, laboratoire des maladies nutritionnelles et d'immunologie des ruminants, \\ Theix, 63122 St-Genès-Champanelle, France
}

(Reçu le 9 octobre 1989; accepté le 4 janvier 1991)

\begin{abstract}
Résumé - Les paramètres du dosage des IgG du lait de vache par immunodiffusion radiale semiautomatisée ônt été étudiés : qualité de la gélose, nature dé l'antisérum et de la gamme d'étalonnage, modalités de diffusion. Les laits sont simplement dilués au $1 / 10$, et des résultats précis peuvent être obtenus après $6-8 \mathrm{~h}$ de diffusion. Dans les conditions optimales, la cadence d'analyse est de 500 échăntillons à l'heure, avec une répétabilité et une reproductibilité satisfaisantes (CV respectifs de 3,2 et $5,1 \%)$. Une bonne corrélation linéaire $(r=0,988)$ a été obtenue avec le dosage par immunonéphélémétrie cinétique. Cette technique ouvre la possibilité d'un contrôle en routine de la qualíté protéique du lait, par le dosage des lgG.
\end{abstract}

IgG bovine / immunodiffusion radiale semi-automatisée / qualité du lait

Summary - Quantification of bovine milk IgG for the detection of colostrum, mastitis milk or milk from late gestation. Parameters of a semi-automatised single radial immunodiffusion technique applied to quantitation of milk IgG have been studied: agar quality, kind of antiserum and standards, diffusion conditions. Milk samples are only diluted to $1 / 10$ and results may be accurately obtained after $6-8 \mathrm{~h}$ diffusion. In optimal conditions milk samples are analysed at the rate of $500 / \mathrm{h}$, with intra and inter-assay $C V$ of 3.2 and $5.1 \%$ respectively. A linear correlation of $r=0.988$ has been obtained with immunonephelometric measurement. This technique permits a systematic control of milk protein quality by $\lg G$ quantification.

bovine IgG / automatised radial immunodiffusion / milk quality 


\section{INTRODUCTION}

La présence, dans les livraisons de lait de vache, de laits anormaux tels que le colostrum, les laits de mammites ou de fin de gestation, est responsable d'une détérioration de la qualité sanitaire, d'une augmentation du taux des protéines solubles et donc d'une baisse de la qualité protéique du lait. Les conséquences économiques en sont importantes : surpaiement du lait, gratinage précoce sur les plaques des pasteurisateurs, baisse du rendement fromager, incidents techniques lors de la fabrication des fromages (retards à la fermentation, égouttage insuffisant des caillés, etc.). Les pertes économiques ont été estimées par Maurice (1979) à $2 \%$ de l'approvisionnement en lait d'une laiterie.

L'apport le plus important de protéines solubles se rencontre lors de l'addition de colostrum, du fait de la richesse extrêmement élevée en immunoglobulines $\mathrm{G}$ ( $\mathrm{lgG}$ ) de cette sécrétion : $60-80 \mathrm{~g} / \mathrm{l}$, pour seulement 0,5-0,6 g/l dans un lait normal (Butler, 1981; Levieux, 1984). Le colostrum contient également des quantités de $\beta$ lactoglobuline et d' $\alpha$-lactalbumine respectivement 3 fois et 2 fois plus élevées que dans un lait normal (Alais, 1984). L'addition de colostrum aux livraisons de lait est interdite par la loi; en effet, aux termes de l'article 2 du décret du 25 mars 1924, modifié et complété par le décret $n^{\circ} 71-6$ du 4 janvier 1971, est considéré comme impropre à la consommation humaine : le lait de vache provenant d'une traite opérée moins de $7 \mathrm{j}$ après le part et, d'une manière générale, le lait contenant du colostrum. Cette législation est rarement respectée à la lettre et la majorité des éleveurs livre généralement le lait dès le $3^{e}$ ou $4^{e}$ jour qui suit le vêlage.

Le lait de mammites est également considéré par le décret du 25 mars 1924 comme impropre à la consommation humaine; il se caractérise par une augmentation du taux des $\lg G$ et, dans une moindre mesure, de l'albumine (Caffin et al, 1983; Poutrel et al, 1983). Les taux de $\beta$ lactoglobuline et d' $\alpha$-lactalbumine sont peu modifiés, généralement dans le sens d'une légère diminution (Caffin et al, 1985).

Dans les laits de fin de gestation, du fait de la préparation de la mamelle à la sécrétion de colostrum, on assiste à une augmentation progressive des protéines solubles essentiellement due à la fraction IgG (Dixon et al, 1961; Kiddy et al, 1971).

La détermination de la teneur en IgG d'un lait de vache est donc, actuellement, le meilleur marqueur de la présence de laits anormaux et d'une augmentation du taux des protéines solubles. Leur dosage par électrophorèse (Maurice, 1979), a vite cédé le pas aux dosages immunologiques, plus simples, plus précis et plus sensibles. Lebreton et al (1981) ont ainsi décrit une technique par immunonéphélémétrie, appropriée aux grandes séries, mais dont le développement a été limité par le prix de revient trop élevé de l'analyse du fait d'une consommation importante d'antisérum et de la nécessité d'un traitement préalable des échantillons incluant leur filtration à $0,2 \mu \mathrm{m}$. L'immunodiffusion radiale (Mancini et al, 1965), plus économe en réactifs et ne nécessitant pas de clarification des échantillons de lait, se prête théoriquement mieux au dosage des lgG; son automatisation permet d'envisager son application aux analyses de grandes séries d'échantillons. Dans cet article, nous définissons les conditions optimales d'application et les performances de cette technique pour le dosage en routine des taux d'lgG du lait de vache, tel qu'il pourrait être appliquée, par exemple, dans le cadre d'un laboratoire interprofessionnel d'analyses laitières. 


\section{MATÉRIEL ET MÉTHODES}

\section{Principe de la technique semi- automatisée d'immunodiffusion radiale, dans son application au dosage des IgG du lait de vache (fig 1)}

Le lait à analyser est déposé dans un puits creusé dans une couche de gélose qui contient un antisérum spécifique des $\lg$ de vache. Au fur et à mesure de la diffusion des protéines du lait dans la gélose, un précipité circulaire se forme entre les $\lg G$ du lait et les anticorps anti-lgG de l'antisérum. La surface du cercle délimité par le précipité est proportionnelle à la concentration en IgG du lait. Pour automatiser le dosage, l'écartement des puits dans la gélose a été standardisé à l'entre-axe $(9 \mathrm{~mm})$ des cupules de plaques de microtitration, matériel couramment utilisé dans les laboratoires interprofessionnels laitiers pour la recherche d'antibiotiques ou d'anticorps contre la leucose bovine. Les laits, disponibles dans ces plaques, sont dilués dans une deuxième plaque puis déposés dans les puits de la gélose par séries de 8 échantillons, grâce à l'utilisation jumelée de 2 diluteurs programmables à 4 canaux, ou d'une micropipette multicanaux du commerce. Après diffusion, les plaques de gélose sont placées sur une boîte d'éclairage à fond noir et l'image des précipités est aggrandie à l'aide d'une caméra vidéo équipée d'un objectif pour macrophotographie. La position des puits sous la caméra est repérée par un support de déplacement $X-Y$, au pas de $9 \mathrm{~mm}$. Un système électronique couplé à un micro-ordinateur analyse le signal vidéo fourni par la caméra et permet la mesure automatique des diamètres en temps réel. Le diamètre effectivement mesuré apparaît en surimpression sur l'image du précipité fournie par le moniteur vidéo, ce qui permet un contrôle visuel permanent. Le micro-ordinateur enregistre le numéro du puits et le diamètre correspondant; il calcule les constantes de l'équation de la droite de régression pour les points de gamme incorporés à chaque série d'analyse, puis, à partir de cette équation, le taux d'lgG de chaque échantillon.

\section{Purification des $\lg G_{1}$ bovines}

Les $\lg _{1}$, principale sous-classe d'IgG présente dans les sécrétions lactées, sont purifiées à partir du colostrum bovin par chromatographie d'échange d'ions et filtration sur gel selon des modalités déjà décrites en détail (Levieux, 1974), puis dialysées contre de l'eau distillée et lyophilisées.

\section{Préparation d'antisérums anti-lg $G_{1}$ bovines}

Des chevaux reçoivent une injection intradermique en points multiples, de $2 \mathrm{ml}$ d'une émulsion à parties égales d'lgG $15 \mathrm{mg} / \mathrm{ml}$ en $\mathrm{NaCl} 0,15$ mol..$^{-1}$ ) et d'adjuvants complet de Freund (Miles). Une deuxième injection est effectuée $45 \mathrm{j}$ plus tard avec la même quantité d'lgG émulsionnée en adjuvant de Freund incomplet (Miles). Les animaux sont prélevés $7 \mathrm{j}$ après la $2^{\mathrm{e}}$ injection. La spécificité des antisérums est contrôlée par immuno-électrophorèse selon Scheidegger (1955), vis-à-vis de sérum et de colostrum bovin. La qualité précipitante des antisérums est testée en immunodiffusion radiale afin de sélectionner les immunsérums de titre élevé en anticorps et qui donnent des disques de précipité à bords bien nets.

\section{Préparation des boîtes pour immunodiffusion radiale}

Dans des boîtes en plastique de $9 \times 6 \mathrm{~cm}$ sont coulés $15 \mathrm{ml}$ d'agar Noble (Difco) à $1,2 \%$ en tampon de Mayer (Biolyon) additionné d'azothydrate de sodium $(0,1 \%)$ et contenant une quantité précise d'antisérum (cf Résultats), suffisante pour obtenir des précipités facilement mesurables. Des puits de diamètre $3,5 \mathrm{~mm}$ et d'entraxe $9 \mathrm{~mm}$ sont pratiqués à l'aide d'un système d'emporte-pièce relié à une pompe à vide. Chaque boîte contient 48 puits, ce qui correspond à une demi-plaque pour microtitration. 

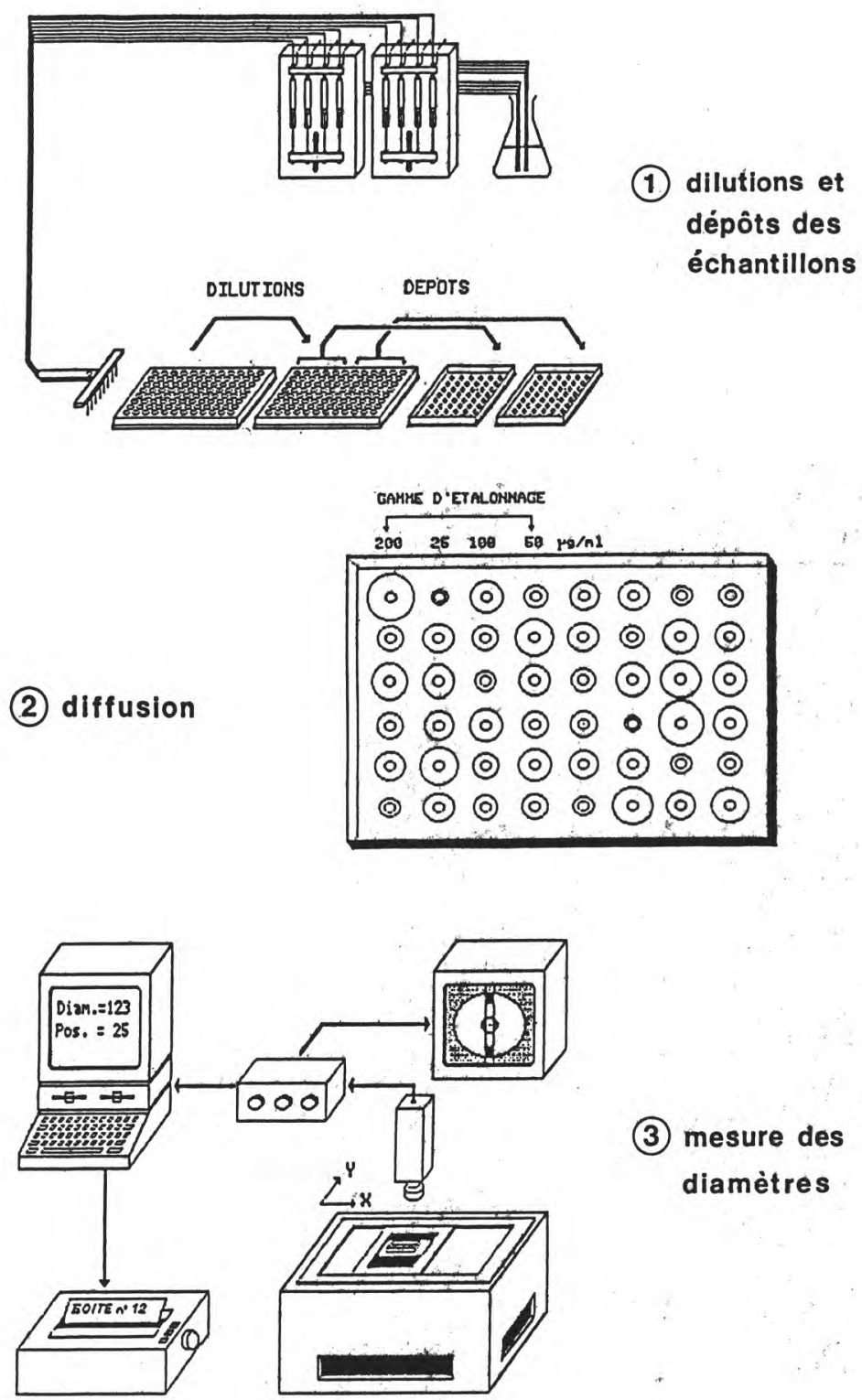

(3) mesure des diamètres

Fig 1. Schéma du principe de la semi-automatisation de l'immunodiffusion radiale. Illustration of the semi-automatized method of radial immunodiffusion. 


\section{Préparation des échantillons}

Les laits frais, conservés une nuit à $+4{ }^{\circ} \mathrm{C}$ ou stabilisés par addition de bichromate de potassium $(0,1 \%)$, ou par congélation à $-20^{\circ} \mathrm{C}$, sont dilués au 1/10 en eau distillée dans une plaque de microtitration à fond plat, sous un volume final de $0,2 \mathrm{ml}$. Les dilutions sont effectuées avec le diluteur programmable Hamilton Microlab MT (Touzart et Matignon) ou une micropipette multicanaux réglable (Dynatech). Les dilutions sont soigneusement homogénéisées sur un agitateur rotatif horizontal (IKA Vibrax VXR). Dans le cas du dosage comparatif par immunodiffusion radiale et immunonéphélémétrie cinétique, le lactosérum est préparé par emprésurage $(0,05 \mathrm{ml}$ de présure renfermant $520 \mathrm{mg}$ de chymosine active par litre) suivi d'une coagulation pendant environ $1 \mathrm{~h}$ a $37^{\circ} \mathrm{C}$ et d'une centrifugation à $4000 \mathrm{~g}$ pendant $20 \mathrm{~min}$. Le lactosérum est prélevé à l'aide d'une seringue munie d'une aiguille, et filtré à $0,22 \mu \mathrm{m}$ au moyen d'un filtre (Millipore) adaptable à l'embout de la seringue.

\section{Immunodiffusion radiate}

Dans les puits des boîtes pour immunodiffusion radiale sont déposés $0,015 \mathrm{ml}$ de chaque échantillon à l'aide du diluteur Hamilton ou de la micropipette multi-canaux. La gamme de référence est constituée par des solutions d'lgG $\mathrm{G}_{1}$ de concentration connue, diluées au $1 / 10$ dans du lait UHT au $1 / 10$ ou dans du tampon de Mayer additionné d'albumine humaine $(0,1 \%)$ et d'azothydrate de sodium $(0,1 \%)$ (cf Résultats) et déposées dans les puits selon les mêmes modalités que pour les échantillons de lait. Les puits non utilisés sont remplis par $0,015 \mathrm{ml}$ d'eau distillée afin de ne pas perturber les conditions de diffusion des échantillons. Les boîtes sont munies de leur couvercle et incuberes à $37^{\circ} \mathrm{C}$ pendant environ $24 \mathrm{~h}$, sur un plan horizontal. L'intensité des précipités est ensuite augmentée en recouvrant la gélose par une solution d'acide acétique à $2 \%$ pendant $1-2$ min puis en rinçant à l'eau distillée pendant 5-10 min.

\section{Lecture des diamètres et calcul des concentrations en IgG}

Les boites sont placées sur le support à déplacement $\mathrm{X}-\mathrm{Y}$ monté sur l'éclairage à fond noir, et les diamètres sont mesurés par un système électronique d'analyse de l'image vidéo couplé au micro-ordinateur Apple lle. Dans les conditions de diffusion précédemment indiquées, le diamètre des précipités est proportionnel à la racine carrée de la concentration en lgG. A partir des points de gamme, l'équation de la régression linéaire est calculée automatiquement et appliquée aux échantillons pour déterminer leur concentration en IgG.

\section{Néphélémétrie cinétique}

L'appareil utilisé est un ICS Beckman; pendant la réaction de précipitátion, il calcule en permanence la dérivée de lintensité du signal (diffractión de la lumière) par rapport au temps. La valeur maximale atteinte par cette dérivée, exprimée en unité arbitraire (Rate Unit), est proportionnelle à la concentration en antigène (IgG dans notre cas) de l'échantillon. Le diluant réactionnel utilisé est une solution de polyéthylèneglycol 6000 à 4,5\% dans du tampon de Mayer (Complement fixation tablets Oxoïd, Biolyon); il donne des résultats équivalents à ceux obtenus avec le diluant Beckman. L'antisérum est dilué (généralement au 1/5) dans ce réactif, mis sous agitation douce pendant 30 min puis centrifugé (10 000 g, $20 \mathrm{~min}$ ). La sensibilité de l'appareil est fixée sur M22. Dans la cuve de mesure, munie d'un mini-barreau magnétique pour l'agitation, sont déposés successivement : $0,6 \mathrm{ml}$ de diluant, $0,04 \mathrm{ml}$ de solutions d'IgG1 ou de lactosérum au $1 / 10$ en tampon de Mayer, puis 0,04 $\mathrm{ml}$ d'antisérum. Le suivi électronique de la réaction est déclenché et la valeur du Rate Unit s'affiche au bout de 30 s en moyenne.

\section{RÉSULTATS}

\section{Boîtes pour immunodiffusion radiale}

Le format des boîtes est imposé par le format des plaques de microtitration à $96 \mathrm{cu}$ pules et par les disponibilités du commerce. Une boîte correspond à la moitié 
d'une plaque de microtitration, et contient 6 rangées de 8 trous au même entre-axe que les cupules des plaques de microtitration. En utilisant 4 points de gamme, il est possible d'analyser 44 échantillons sur la même boîte. Le diamètre des trous $(3,5$ $\mathrm{mm}$ ) permet de déposer commodément $0,015 \mathrm{ml}$ de lait dilué, volume qui peut être délivré avec une bonne précision, sans risques de débordement.

Différentes qualités d'agar-agar et d'agarose sont comparées pour le dosage des lgG du lait de vache. Peu de différences sont observées au niveau de la transparence, pour une concentration de $1,2 \%$, par contre les qualités précipitantes sont extrêmement variables (tableau I) : l'Agar Noble et le Bacto Agar Difco donnent les précipités les plus intenses tandis que les 2 lots d'Agarose (Indubiose IBF et Agarose C Pharmacia) donnent, en l'absence d'acide acétique, des précipités peu intenses mais à bords très nets, et, en présence d'acide acétique, des précipités à peine visibles.

La nature du diluant de la gélose est un paramètre moins critique; des précipités légèrement plus intenses sont cependant obtenus en tampon de Mayer, par rapport à l'eau distillée.

La concentration de l'antisérum est ajustée de façon à obtenir des précipités facilement mesurables dans une gamme d'lgG de 0,025 à $0,4 \mathrm{~g} / \mathrm{l}$, gamme qui couvre l'essentiel des teneurs que l'on peut rencontrer dans des laits de mélange dilués au $1 / 10$. Cette concentration varie en fonction des lots d'antisérums, elle est de l'ordre de $0,1 \mathrm{ml}$ pour $15 \mathrm{ml}$ de gélose.

La conservation des boîtes d'immunodiffusion radiale, testée pendant 4 mois sur des boîtes préparées au laboratoire est excellente, si l'on respecte les conditions suivantes: stockage à $4^{\circ} \mathrm{C}$, sur une surface strictement horizontale et dans un emballage étanche ou dans une chambre humide (récipient à couvercle étanche, muni de couches de papier saturées d'eau).

\section{Gamme d'étalonnage}

Deux types de gamme sont comparés : d'une part, des $\lg _{1}$ bovines purifiées et lyophilisées, mises en solution dans du tampon de Mayer additionné d'albumine humaine $(0,1 \%)$ et d'azothydrate de sodium $(0,1 \%)$ et, d'autre part, du sérocolostrum bovin à $90 \mathrm{~g} / \mathrm{l}$ d'lgG $\mathrm{G}_{1}$ dilué dans du lait entier UHT et stabilisé par du bichromate de potassium $(0,1 \%)$. Ces gammes sont ensuite diluées au 1/10 dans les mêmes conditions que les échantillons de lait. Après $24 \mathrm{~h}$ de diffusion, on ne note pas de différence significative entre les 2 types de gamme. Une disposition alternée des points de gamme, par exemple 0,3$0,02-0,2-0,05-0,1 \mathrm{~g} / \mathrm{l} \mathrm{d}^{\prime} \mathrm{lgG}_{1}$, évite la coa-

Tableau I. Influence de la nature du support d'immunodiffusion radiale sur l'intensité de la précipitation des IgG par une même concentration d'antisérum, évaluée qualitativement de + (précipité à peine visible) à ++++ (précipité très intense).

Influence of the type of support medium used in radial immunodiffusion on the intensity of the precipitation of $\lg G$ for similar concentrations of antiserum, determined quantitatively from + (faint precipitate) to ++++ (dense precipitate).

Intensité du précipité

$\begin{array}{lc}\text { Agar Noble (Difco) } & ++++ \\ \text { Bacto agar (Difco) } & +++ \\ \text { Indubiose (IBF) } & + \\ \text { Agar agar (Merck) } & ++ \\ \text { Agar agar (Touzart et Matignon) } & ++ \\ \text { Agarose C (Pharmacia) } & +\end{array}$


lescence partielle des précipités de grands diamètres, ce qui facilite la mesure. La relation entre le diamètre et la racine carrée de la concentration en IgG est linéaire entre 0,02 et $0,3 \mathrm{~g} / \mathrm{l}$, après 18 à $24 \mathrm{~h}$ de diffusion à $37^{\circ} \mathrm{C}$.

\section{Préparation et dépôt des échantillons de lait}

Les échantillons de lait ne subissent qu'un seul traitement : une dilution au 1/10 en eau distillée. Dans la mesure où les laits sont déjà disponibles dans les plaques de microtitration à 96 cupules, cette dilution dans une $2^{\mathrm{e}}$ plaque puis le dépôt de la dilution dans les puits de la gélose est simple et rapide : 5 min par plaque de 96 cupules, soit environ 1000 dilutions et dépôts à l'heure. L'utilisation du diluteur Microlab MT pour la dilution et le dépôt des échantillons est légèrement plus précise (cf tests de répétabilité) et plus confortable pour le manipulateur que la manipulation de la micropipette multicanaux.

\section{Diffusion}

Différents temps de diffusion ont été testés, pour une incubation à température constante $\left(37^{\circ} \mathrm{C}\right)$. II est possible de mesurer les diamètres des précipités dès la $4^{\mathrm{e}} \mathrm{h}$ de diffusion (fig 2), cependant la sensibilité est faible, ce qui réduit la précision de l'analyse. Après 6-8 h de diffusion, un dosage plus précis est possible, bien que la relation entre le diamètre et la racine carrée de la concentration ne soit pas linéaire sur toute la gamme; la linéarité complète n'est obtenue qu'après 18-24 h de diffusion. Les boîtes munies de leur couvercle et incubées dans une étuve à $37^{\circ} \mathrm{C}$, ou placées dans l'étuve, sans leur couvercle mais dans une chambre humide, donnent des résultats strictement comparables.

\section{Mesure des diamètres}

Cette mesure peut s'effectuer sur l'écran vidéo, à l'aide d'un double décimètre : la cadence est d'environ 250 échantillons à l'heure, indépendamment du temps de calcul. Avec le système de seuillage en temps réel du signal vidéo, couplé au micro-ordinateur, l'analyse complète (mesures, calculs, impression de la courbe d'étalonnage et des taux d'lgG) de 1000

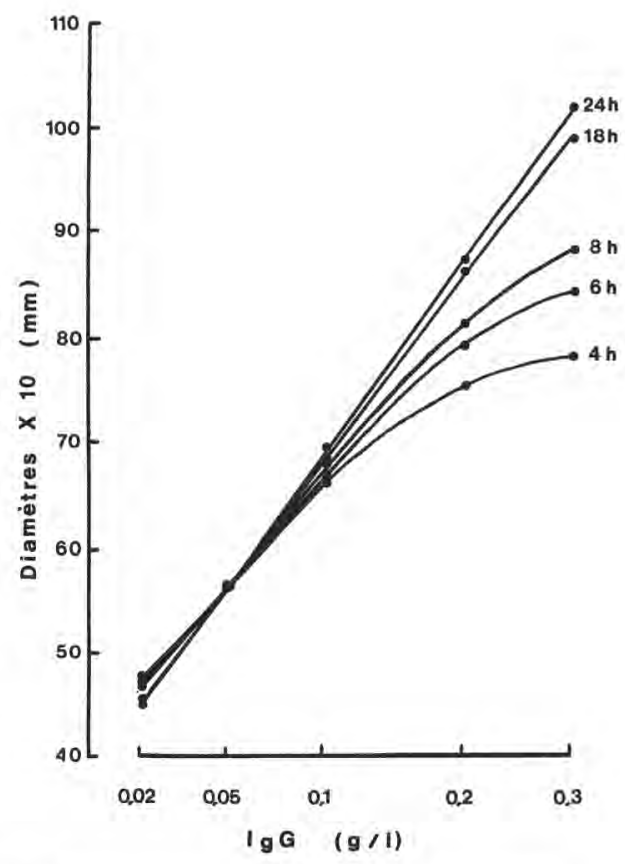

Fig 2. Courbes d'étalonnage obtenues en immunodiffusion radiale semi-automatisée après différents temps de diffusion à $37^{\circ} \mathrm{C}$. L'axe des $X$ est gradué en portant la valeur de la racine carrée de la concentration en $\lg G$ sur une échelle arithmétique.

Standard curves obtained from semiautomatized method of radial immunodiffusion after different diffusion times at $37^{\circ} \mathrm{C}$. The $\mathrm{X}$ axis is graduated using the square root value of IgG concentrations on an arithmetic scale. 
échantillons est réalisée en $1 \mathrm{~h}$, la mesure des diamètres représentant environ la moitié de ce temps.

\section{Répétabilité}

Elle est fonction de la qualité des dilutions et du dépôt des échantillons de lait, de la bonne homogénéité de l'antisérum dans la gélose et de la constance de l'épaisseur de celle-ci, et de la précision de la mesure des diamètres.

Pour la qualité des dilutions et du dépôt des échantillons, sont comparés la micropipette multicanaux et le diluteur programmable Hamilton Microlab MT, les autres paramètres étant considérés comme des constantes, notamment la mesure automatique des diamètres qui s'effectue avec une précision d'une ligne vidéo, pour des diamètres généralement compris entre 100 et 250 lignes. Les résultats obtenus à partir du dosage, répété 4 fois, d'un lait de grand mélange déposé dans 44 cupules d'une même boîte sont présentés sur le tableau II. On constate une nette supériorité des dosages effectués à l'aide du diluteur programmable : coefficient de variation de $3,2 \%$ contre $6,6 \%$, en moyenne, avec la micropipette multicanaux.

Lorsque des trous dans la gélose ne sont pas remplis de liquide, on observe une modification de la diffusion des échantillons de lait avoisinants : les précipités sont légèrement ovales et leur diamètre moyen est augmenté d'environ $5 \%$, ce qui surestime le taux d'IgG d'environ 15-20\%. II est donc impératif de remplir avec 0,015 $\mathrm{ml}$ d'eau tous les trous non utilisés par des échantillons.

\section{Reproductibilité}

Elle a été testée sur 44 échantillons de laits analysés 5 fois à 24 h d'intervalle. Les résultats, présentés dans le tableau II, confirment la plus grande précision des dilutions effectuées avec le diluteur automatique : $5,1 \%$ contre $7,2 \%$ avec la pipette multicanaux.

\section{Cadence d'analyse}

À partir d'échantillons de laits disponibles dans des plaques de microtitration (ce qui est le cas dans de nombreux laboratoires interprofessionnels laitiers), et en ne comptant pas les $24 \mathrm{~h}$ de diffusion dans l'étuve à $37^{\circ} \mathrm{C}$, la cadence d'analyse est de 500 échantillons à l'heure, y compris le temps de calcul, d'impression des gammes d'étalonnage et des tableaux de résultats.

\section{Comparaison du dosage des IgG par immunonéphélémétrie et immunodiffusion radiale}

Un échantillonnage de 44 laits individuels, couvrant des taux d'lgG compris entre 0,2 et $1,5 \mathrm{~g} / \mathrm{l}$, a été analysé par les 2 techni-

Tableau II. Coefficients de variation obtenus dans les tests de répétabilité intra-boîte (4 boîtes recevant chacune 44 échantillons de lait identiques) et de reproductibilité (44 échantillons différents analysés 5 fois à $24 \mathrm{~h}$ d'intervalle).

Variation coefficient obtained for intra-box repeatability (4 boxes each receiving 44 identical milk samples) and for reproducibity (44 different samples analysed 5 times over a 24-h period).

\section{Diluteur}

Programmable Manuel

Répétabilité (CV \%)

3,2

Reproductibilité (CV \%)
6,6

7,2 
ques (fig 3). La droite de régression de $Y$ (immunonéphélémétrie) en $X$ (immunodiffusion radiale) a pour équation : $Y=1,04 X$ - 0,028. Le coefficient de corrélation linéaire calculé pour cette droite est : $r=$ 0,988 .

\section{DISCUSSION}

Les techniques de précipitation en gélose, telle l'immunodiffusion radiale, ont un grand avantage sur les techniques de précipitation en milieu liquide comme la néphélémétrie : elles ne sont pas dépendantes de la turbidité propre des échantillons. Dans le cas du lait, c'est un atout essentiel puisqu'il n'est pas nécessaire de coaguler individuellement les échantillons puis de filtrer à $0,2 \mu \mathrm{m}$ les lac-

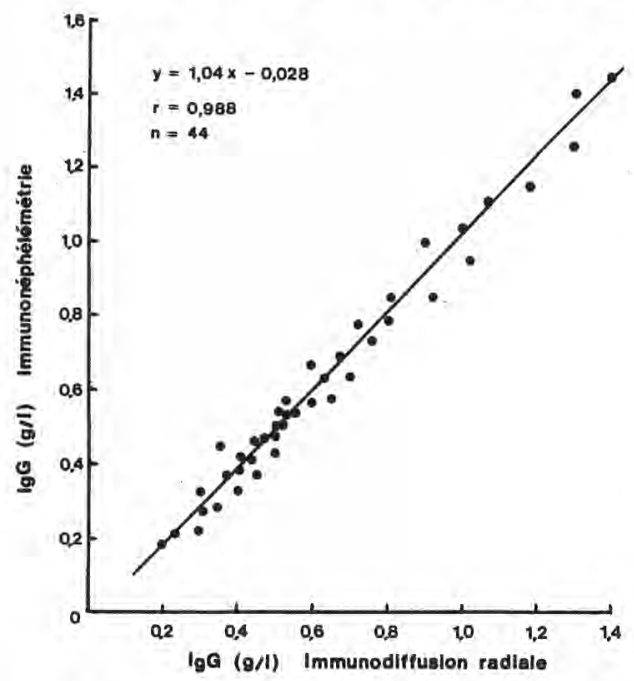

Fig 3. Corrélation entre le dosage des lgG du lait de vache par immunodiffusion radiale semiautomatisée et immunonéphélémétrie cinétique obtenue sur 44 échantillons de laits individuels.

Correlation between the measurement of cow milk IgG by semi-automatized radial immunodiffusion and rate nephelometry obtained with 44 different milk samples. tosérums obtenus. Ces opérations, décrites en détail par Lebreton et al (1981) pour le dosage immunonéphélémétrique des IgG du lait, sont coûteuses en maind'cuvre et en produits; de ce fait, elles limitent les possibilités d'application en routine du dosage des lgG dans le cadre d'un paiement multi-critères du lait. Dans les modalités précédemment décrites, l'immunodiffusion radiale semi-automatisée ne nécessite qu'une dilution du lait au 1/10, réalisable en une seule étape qui, outre la simplicité, permet d'obtenir des résultats directement exprimés en g par litre de lait et non de lactosérum, ce qui évite d'avoir à corriger les résultats par un facteur variable en fonction de la composition physico-chimique de chaque échantillon; par ailleurs, elle consomme environ 4 fois moins d'antisérum, réactif particulièrement coûteux. Ces éléments concourent à réduire approximativement d'un facteur 4 le prix de revient d'une analyse qui, bien entendu, est fonction de l'importance des séries de dosage.

L'immunonéphélémétrie présente l'avantage de pouvoir donner un résultat dans I'heure qui suit l'arrivée de l'échantillon au laboratoire; dans le cadre d'un contrôle de routine de la qualité du lait, cette rapidité de réponse n'est cependant pas impérative. II faut par ailleurs rappeler qu'une lecture des plaques d'immunodiffusion radiale est possible, grâce à la révélation par l'acide acétique, dès la $4^{\mathrm{e}} \mathrm{h}$ de diffusion, bien que la précision optimale soit obtenue après $18-24 \mathrm{~h}$ de diffusion. Ces conditions sont très éloignées de celles décrites par Lebreton et al (1981) : diffusion de $48 \mathrm{~h}$ suivie de $3 \mathrm{j}$ de lavage et d'une coloration au Bleu de Coomassie précédant la mesure des diamètres. L'utilisation d'une concentration d'agar plus faible $(1,2 \%$ au lieu de $2,5 \%$ ), la diffusion à $37^{\circ} \mathrm{C}$, la révélation par l'acide acétique et la mesure des diamètres à l'aide d'une caméra vidéo 
nous a permis de ramener ce temps d'analyse à un maximum de $24 \mathrm{~h}$ tout en diminuant la quantité d'antisérum requise.

L'immunodiffusion radiale présente, par contre, un avantage analytique important sur l'immunonéphélémétrie : elle n'induit pas le manipulateur en erreur dans les cas d'excès d'antigène, c'est-à-dire lorsque les taux d'lgG sont anormalement élevés. Dans les conditions décrites par Lebreton et al (1981) lorsque les taux d'IgG dépassent $2,5 \mathrm{~g} / \mathrm{l}$, la quantité de précipité obtenue atteint un plateau (zone d'équivalence) puis diminue. Ainsi, pour une concentration en $\mathrm{lgG}$ de $10 \mathrm{~g} / \mathrm{l}$ le signal donné par le néphélémètre devient le même que celui obtenu pour un échantillon à environ $1 \mathrm{~g} / \mathrm{l}$. De telles concentrations peuvent se rencontrer lors de l'addition de colostrums très riches en IgG (des taux d'lgG supérieurs à $200 \mathrm{~g} / \mathrm{l}$ sont fréquemment observés) ou lors de l'analyse de laits individuels prélevés dans les 4-5 j qui suivent le vêlage ou provenant de vaches en fin de gestation. En immunodiffusion radiale, de telles concentrations donnent des précipités dont les diamètres sont encore proportionnels à la concentration en lgG, selon une régression non plus linéaire mais polynomiale d'ordre 3 . La très grande taille de ces diamètres alerte l'opérateur qui peut alors décider d'analyser les échantillons à une dilution plus élevée.

L'immunodiffusion radiale est, par ailleurs, une technique plus rustique que l'immunonéphélémétrie : la qualité précipitante des antisérums est moins déterminante, et de nombreux lots d'antisérums inutilisables en immunonéphélémétrie peuvent être utilisés en immunodiffusion radiale tandis que l'inverse n'est, à notre connaissance, jamais observé; d'autre part, la nature du milieu réactionnel, qui peut légèrement varier selon la composition physico-chimique de l'échantillon, mo- difie beaucoup plus facilement la précipitation en milieu liquide que la précipitation en milieu gélosé. Ceci explique certainement les différences de résultats observés par Lebreton et al (1981) lorsque le même échantillon de lait est analysé pur, dilué au $1 / 2$ ou au $1 / 4$.

Contrairement aux résultats publiés par Lebreton et al (1981), nous observons un bon coefficient de corrélation linéaire entre les dosages effectués par les 2 techniques appliquées aux mêmes échantillons de lactosérums $(r=0,988$ au lieu de 0,90$)$ et, surtout, des concentrations équivalentes d'IgG au lieu d'une surestimation de l'ordre de $50 \%$ par l'immunodiffusion radiale. Une première explication à ces divergences de résultats peut se trouver dans la différence de matériel utilisé pour l'immunonéphélémétrie : le néphélémètre cinétique (Beckman) que nous utilisons mesure la lumière diffractée à un angle différent de celui utilisé dans le néphélémètre Behring utilisé par ces auteurs. II est, par ailleurs, moins sensible à la présence de particules dans le milieu réactionnel puisqu'il mesure la vitesse de formation du précipité, et non la quantité de précipité à un instant donné; or, il est difficile de garantir la parfaite efficacité de la filtration des lactosérums en grande série ou l'absence de quelques particules dans le diluant dans la cuve de mesure (avant ou pendant la réaction), ce qui réduit la fiabilité de la néphélémétrie en point final. En témoignent les «accidents" observables dans la courbe d'équivalence néphélémétrique publiée par ces auteurs (signal pratiquement identique obtenu pour 0,32 ou $0,64 \mathrm{~g} / \mathrm{l}$ d'lgG purifiées), "accidents" que l'on ne retrouve pas dans leur courbe d'équivalence en tube. Une deuxième explication tient à la différence de nature de l'antisérum et de gamme utilisées. Les lgG du lait de vache sont essentiellement des $\lg _{1}$, la sous-classe $\lg _{2}$ étant présente en concentration 10 fois 
plus faible (Butler, 1981); nous avons donc choisi de préparer un antisérum dirigé essentiellement contre les $\lg G_{1}$, en immunisant les animaux avec des $\lg _{1}$ purifiées à partir de colostrum, et d'utiliser ces mêmes $\operatorname{lgG}_{1}$ pour la gamme d'étalonnage. Lebreton et al (1981), ont préparé un antisérum contre une préparation d'lgG $\mathrm{G}_{2}$ purifiées à partir du sérum sanguin, et utilisé ces $\lg G_{2}$ pour la gamme d'étalonnage. En conséquence, ils observent 2 systèmes précipitants distincts en immunodiffusion radiale, correspondant aux $\operatorname{lgG}_{1}$ et $\operatorname{lgG}_{2}$ phénomène qui indique la présence dans l'antisérum d'une concentration importante d'anticorps dirigés contre les déterminants spécifiques des $\lg G_{2}$, anticorps qui précipiteront en grande quantité avec les $\lg _{2}$ de la gamme d'étalonnage, mais qui seront par contre peu impliqués lors du dosage des lgG du lactosérum. Bockhout (1975) a ainsi montré que des analyses effectuées avec des antisérums non appropriés peuvent donner des taux d'lgG double du taux réel. Pour le contrôle de la qualité du lait de vache par dosage des IgG, il est donc impératif que l'antisérum et la gamme d'étalonnage soient préparés à partir d'lgG $G_{1}$ bovines purifiées, et non à partir de solutions de "gamma-globulines" du commerce qui sont obtenues à partir de sérums sanguins de bovins et contiennent essentiellement des $\lg \mathrm{G}_{2}$.

En conclusion, avec les améliorations apportées sur le plan de l'automatisation, et dans les conditions expérimentales que nous avons mises au point pour l'analyse d'échantillons de lait, l'immunodiffusion radiale permet un dosage simple, spécifique et reproductible des IgG, pour une cadence et un prix de revient de l'analyse compatibles avec les exigences d'un contrôle de routine appliqué dans le cadre d'un paiement multi-critères du lait.

\section{REMERCIEMENTS}

Nous tenons à remercier $M$ Retière, directeur du LIAL (18800 Aurillac), pour l'intérêt soutenu qu'il a porté à ce travail, M Lapied, directeur du LABILAIT (76230 Bois-Guillaume) pour les informations qu'il nous a apportées sur sa grande pratique de l'immunonéphélémétrie appliquée en routine dans le cadre d'un laboratoire interprofessionnel laitier, et la Société Immuno-Centre (4 rue $P$ Eluard, 58640 Varennes-Vauzelles) pour la fourniture gracieuse de kits de dosage des IgG du lait. Nous remercions également Melle Devins pour son excellente assistance technique.

\section{RÉFÉRENCES}

Alais C (1984) Science du lait, principes des techniques laitières. $4^{e}$ édition, Sepaic, Paris

Bockhout BA (1975) Divergent results in radial immunodiffusion with antisera differing in precipitation properties with respect to individual immunoglobulin subclasses. I. The antigen factor. II. The antiserum factor. $J$ Immunol Methods 7, 187-210

Butler JE (1981) A concept of humoral immunity among ruminants and an approach to its investigation. In: The Ruminant Immune System (JE Butler, ed) Plenum Press, NY, 3-55

Caffin JP, Poutrel B, Rainard P (1983) Physiological and pathological factors influencing bovine immunoglobulin $G_{1}$ concentration in milk. J Dairy Sci 66, 2161-2166

Caffin JP, Poutrel B, Rainard P (1985) Physiological and pathological factors influencing bovine $\alpha$-lactalbumin and $\beta$-lactoglobulin concentrations in milk. J Dairy Sci 68, 10871094

Dixon FJ, Weigle WO, Vazquez JJ (1961) Metabolism and mammary secretion of serum proteins in the cow. Lab Invest 10, 216-236

Kiddy CA, McCann R, Maxwell C, Rock C, Pierce C, Butler JE (1971) Changes in levels of immunoglobulins in serum and other body 
fluids immediately before and after parturition. J Dairy Sci 60, 546-556

Lebreton JP, Joisel F, Boutleux S, Lannuzel B, Sauger $F$ (1981) Dosage immunonéphélométrique des immunoglobulines $\mathrm{G}$ dans le lait de vache. I. Mise au point technique du dosage. Lait 61, 465-480

Levieux D (1974) Immunoglobulines bovines et brucellose. I. Purification des immunoglobulines et préparation de leurs antisérums spécifiques. Ann Rech Vet 9, 343-353

Levieux D (1984) Transmission de l'immunité passive colostrale. In: Physiologie et Pathologie Périnatale chez les Animaux de Ferme. INRA Publ Versailles, 21-30
Mancini G, Carbonara AO, Heremans JM (1965) Immunological quantitation of antigens by single radial immunodiffusion. Immunochemistry 2, 235-254

Maurice L (1979) Dosage électrophorétique des lgG du lait de vache. Mémoire, École supérieure des ingénieurs et techniciens en alimentation. Le Vaudreuil 27100, France

Poutrel B, Caffin JP, Rainard P (1983) Physiological and pathological factors influencing bovine serum albumin content of milk. J Dairy Sci 66, 535-541

Scheidegger JJ (1955) Une microméthode de l'immunoélectrophorèse. Int Arch Allergy Appl Immunol 7, 103-111 